\title{
NAGI METHODS OF MAP QUALITY EVALUATION
}

\author{
Dr. Nagi Zomrawi \\ Associate Professor, College Engineering, Sudan University of Science and Technology, \\ Sudan, Khartoum, P.O Box: 407
}

\begin{abstract}
Map quality evaluation is one of the important tasks that can face map specialists in particular and map users in general. Numerical description of the map product to justify its quality was studied by the author who has developed before, a new approach of map quality evaluation that, successfully applied globally by different researchers. Here, in this research work, a new formula has been developed to numerically evaluate map product. The evaluation base on computing a satisfaction factor suggested to be called Weighted Nagi Factor. The developed formula assumes different weights of the map criteria according to map application or specification adopted. This new Weighted Nagi Method, successfully applied to a sample map product of Khartoum state survey authority. Results proved that the new weighted method is practicable to numerically describe a map product taking into account the importance of each criteria adopted according to a particular application. The method is also simple and easy to be applied by any stakeholder, map quality evaluator or map user.
\end{abstract}

KEYWORDS: Average, Map standards, Nagi Factor, Quality assurance, Quality control, Weight, Weighted average, Weighted Nagi-Factor.

\section{INTRODUCTION}

In year 2013, author has developed a new approach of map quality evaluation according to existence and satisfaction of a map product to required mapping criteria and successfully examined it.

That new approach (or call it Nagi Method of Map Quality Evaluation) was simply based on identifying the suggested map requirements and their criteria according to specification or map standards. Then, applying evaluation criteria to the map product under evaluation. As a result, three cases may be expected. First; existence of the map requirement according to the specification criteria, i.e. satisfying the map standards. Second; existence of the map requirement but, unsatisfying the map standards. Third; the complete absence of the map requirement. In order to numerically describe these three cases, 2, 1 and 0 points were suggested to be given to each case successively.

Final evaluation can be done by computing a satisfaction factor suggested to be called $N$-Factor or Nagi-Factor. This factor is a percentage ratio of the summation of the map points to the points of the ideal case, i.e. twice the number of evaluation criteria used.

Mathematical model of this method can be formulated as:

$$
\text { Nagi Factor }=\frac{\sum p}{2 \times n} \times 100 \%
$$

Where, 
$p$ is the evaluation points given for each criteria,

$n$ is the number of criteria applied to the map.

Acceptance or rejection of the map product base on the value of Nagi Factor that assigned by mapping quality desired.

In order to apply that new approach, number of evaluation criteria were selected and adopted to a sample map (part of Khartoum State - Sudan). Then, N-Factor (Nagi Factor) was computed according to Sudan map specification ${ }^{[7]}$.

Fortunately, this new approach found a good reputation and response. It was successfully applied in different areas such as Tamil Nadu State Survey Authority map product (India) ${ }^{[10]}$.

That new developed approach proved to be simple, easy and applicable by even non specialist [7].

\section{Mapping Technology}

Map, as conventionally defined, is a graphical (symbolized) representation of geospatial data, that is refer to the location or the attributes of object or phenomena location on the earth. Map helps users to better understand geospatial relationship and provides information of distance, direction and area.

Maps should be simple, clear and convey information efficiently. Its colours and overlays must be legible to the user.

Maps should satisfy the basic elements that include title, legend, scale, projection, north direction, locality map, grid system, map index and preparation date. These elements and other may depend on the specification of the mapping agency ${ }^{[7]}$.

Mapping technologies have been developed during the time. In the past, mapping process was considered to be hard and tedious, that is because of the old methods and conventional equipment that have been used such as, chain, campus, plane table, optical level and theodolite. Such survey tools required a number of persons to be in the job and time consuming to achieve the desired accuracy, rather than the accommodation and the team camp facilities required. Modern instruments such as total station and Global Positioning System (GPS) may speed the time of data collection and reduce the cost to some extent. However, still land survey equipment are slow and cost effective for mapping large areas. Therefore, photogrammetry could be a practical substitute for mapping large areas.

Photogrammetric techniques based on covering the area by successive overlapping photographs in parallel flightstrips. Then, maps could be produced utilizing photographs rather than collecting data directly from the field. Thus reducing the time and minimizing the cost.

Recently, satellite remote sensing becomes an attractive tool of collecting mapping data because, it provides number of advantages. These advantages may include large data coverage, accessibility, continuity and homogeneity of data. Moreover, it provides a low cost means of data collection. These advantages of remote sensing over the other mapping data collection techniques make it an attractive tool in different fields. Today, remote sensing affords a practical means of frequent and accurate monitoring of the earth's resources on literally a global basis. It is aiding in assessing the impact of human activities on air, water and land. Data 
obtained from remote sensors have provided information necessary for making sound decisions and formulating policy in a host of resource-development and land use applications ${ }^{[8]}$.

\section{Quality Control and Quality Assurance}

Quality control (QC) can be defined as the set of activities performed to ensure that the products or services meet the requirements as defined during the earlier process for assurance of quality.

Assurance of quality is a prevention system that predicts almost everything about product safety, quality standards and legality that could possibly go wrong and then takes steps to control and prevent flawed products or services from reaching the advanced stages of the supply chain.

Quality Assurance (QA) processes involve implementing real-time quality checks in every department of the company like designing, research, manufacturing and marketing.

QA/QC are an integral factors in the data collection task and cannot be sacrificed to expediency or false economy.

A relatively limited QA/QC effort is usually directed toward field tests to verify the quality of final mapping products. As a consequence, credible judgment must be exercised by both mapper and user in designing a mapping project so that the accuracy of the final output adheres to reliable tolerances.

Quality control should be the watchword of the mapper who is obligated to institute professional quality control procedures. Quality assurance should be ascertained by the user who is committed to performing quality assurance assessment measures ${ }^{[11]}$.

\section{Statistical Concepts}

Measure of central tendency is a statistical value used to describe numerically a data set. Average or arthematic mean is usually used to describe a set of data. Where, other measures such as median, mode, geometrical mean, quartiles, deciles and percentiles can also be used.

The average for the set of values $x_{1}, x_{2}, x_{3}, \ldots, x_{n}$ (denoted by $\bar{x}$ ) is the sum of these values divided by their number $(n)$; or:

$$
\bar{x}=\frac{\sum_{i=1}^{n} x_{i}}{n}
$$

Weighting, is statistical technique in which a data item (such as an average) is emphasized more than other data items comprising a group or summary. A number (weight) is assigned to each data item that reflects its relative importance based on the objective of the data collection [4].

Weighted average is the computed average, using the weighting factor, for each data item i.e.

$$
\text { weighted average }=\frac{w_{1} \cdot x_{1}+w_{2} \cdot x_{2}+\cdots+w_{n} \cdot x_{n}}{w_{1}+w_{2}+\cdots+w_{n}}
$$

or; 


$$
\text { weighted average }=\frac{\sum_{i=1}^{n} w_{i} \times x_{i}}{\sum_{i=1}^{n} w_{i}}
$$

Where,

$x_{1}, x_{2}, \ldots x_{n}$ are data set of $n$ items, and

$w_{1}, w_{2}, \ldots w_{n}$ are the weights of each item of the data set.

\section{Weighted NAGI Method of Map Quality Evaluation}

Taking reliable measurements from a map, may be one of important issues of some map applications. Therefore, in such particular application, some map criteria like correct scale and horizontal or vertical accuracy, may appeared to be more important than other criteria. This thing leads the author to improve that developed NAGI METHOD of map quality evaluation to this new approach (suggested to be called WEIGHTED NAGI METHOD of map quality evaluation) by giving a suitable weight for each criteria according to application requirements. Therefore, the new evaluation method based on identifying the suggested map requirements and their criteria first according to specification. Then, giving suitable weight for each required criteria according to application of the map or specification adopted.

During the evaluation steps, map product has to be examined and evaluation criteria applied to justify the existence of the map requirement according to the specification criteria, i.e. satisfaction of the map standards; or not. Here, three cases may arise;

i. Map producer may apply the specification criteria and satisfy its requirement,

ii. Map producer may apply the specification criteria but does not satisfy its requirement, and

iii. Map producer may doesn't apply the specification criteria at all.

Numerical evaluation for each of above mentioned cases can assigned by giving 2, 1 and 0 points for each case successively.

Final evaluation can be done by computing a factor suggested to be called Weighted Nagi Factor. This factor is half the percentage weighted average of the map points, or;

$$
\text { Weighted Nagi Factor }=\frac{1}{2} \frac{\sum_{i=1}^{n} w_{i} \times p_{i}}{\sum_{i=1}^{n} w_{i}} \times 100 \%
$$

Where,

$w_{1}, w_{2}, \ldots w_{n}$ are the weights of each criteria,

$p_{i}$ is the points given for each criteria,

According to the result, acceptance or rejection of the map product can be based on the value of the computed map Weighted Nagi Factor compared with that value assigned by the map standards of the mapping agency. 
Figure (1) below demonstrates applications steps of Weighted Nagi Method of map quality evaluation.

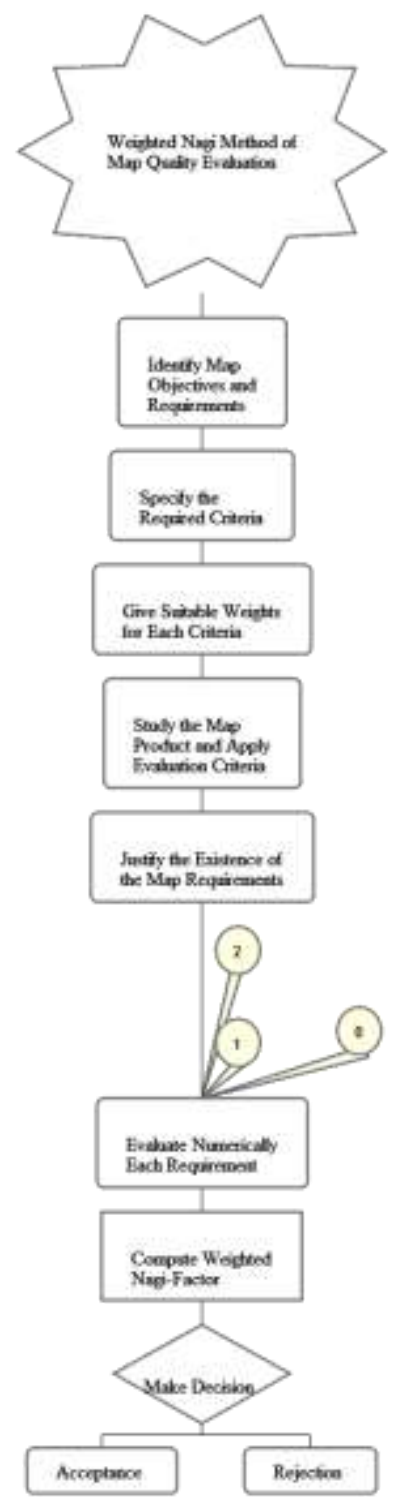

Figure:1 Application steps of Weighted Nagi Method of map quality evaluation.

\section{Measurements and Results}

This work is an extension of a previous approach that developed as a new method of map quality evaluation. The previous Nagi method assumes all map criteria of equal weights. Khartoum survey authority maps products was evaluated using Nagi method subject to Sudan National Survey Authority Standards. In order to do this, a part of Khartoum map covering Kafory block3 was selected to be a sample map. Evaluation criteria of eighteen map elements were specified, examined and evaluated as shown in table (1) below. 
Table:1 Nagi Method.

\begin{tabular}{|c|c|c|}
\hline No. & Evaluation criteria & $\begin{array}{c}\text { Points } \\
(p)\end{array}$ \\
\hline 1 & Scale Accuracy & 0 \\
\hline 2 & Map Title & 2 \\
\hline 3 & North Arrow & 2 \\
\hline 4 & Scale & 2 \\
\hline 5 & legend & 1 \\
\hline 6 & Georeference & 1 \\
\hline 7 & Colour & 1 \\
\hline 8 & sheet Number & 0 \\
\hline 9 & Map Index & 1 \\
\hline 10 & Grid & 0 \\
\hline 11 & Overlapping & 1 \\
\hline 12 & Reliability & 1 \\
\hline 13 & Elevation & 0 \\
\hline 14 & Glossary & 0 \\
\hline 15 & Conversion of elevation & 0 \\
\hline 16 & Magnetic declination & 0 \\
\hline 17 & Lettering & 1 \\
\hline 18 & Source of data & 0 \\
\hline & Sum $(p)$ & 13 \\
\hline & $2 \times n$ & 36 \\
\hline \multicolumn{2}{|r|}{ Nagi Factor $=(13 / 18) \times 100 \%$} & $36 \%$ \\
\hline
\end{tabular}

By applying Nagi method of map quality evaluation, the satisfaction factor that suggested to be called Nagi Factor can computed to be $36 \%$ as shown in the table above.

Now, as discussed, by giving different weights to evaluation criteria, weighted Nagi Method can be applied and new evaluation can be obtained.

Assuming that the main issue of the map product is to take planimetric measurement from. Therefore, some map elements should have weights greater than other.

Table (2) below represents suggested weights for the eighteen evaluation criteria used above. 
Table:2 Suggested weights of map criteria.

\begin{tabular}{||c|l|c||c|l|c||}
\hline \hline No. & Evaluation criteria & $\left(\boldsymbol{w}_{\boldsymbol{i}}\right)$ & No. & Evaluation criteria & $\left(\boldsymbol{w}_{\boldsymbol{i}}\right)$ \\
\hline \hline 1 & Scale Accuracy & 2 & 10 & Grid & 1 \\
\hline 2 & Map Title & 1 & 11 & Overlapping & 1 \\
\hline 3 & North Arrow & 1 & 12 & Reliability & 2 \\
\hline 4 & Scale & 1 & 14 & Elevation & 1 \\
\hline 5 & legend & 2 & 15 & $\begin{array}{l}\text { Conversion of } \\
\text { elevation }\end{array}$ & 1 \\
\hline 6 & Georeference & 1 & 16 & $\begin{array}{l}\text { Magnetic } \\
\text { declination }\end{array}$ & 1 \\
\hline 7 & Colour & 1 & 17 & Lettering & 1 \\
\hline 8 & sheet Number & 1 & 18 & Source of data & 2 \\
\hline 9 & Map Index & \multicolumn{2}{|l}{}
\end{tabular}

Applying the new Weighted Nagi Method and computing Weighted Nagi Factor, result can be obtained as $37 \%$. This result can be demonstrated as shown in table (3) hereunder.

Table:3 Weighted Nagi Method.

\begin{tabular}{|c|c|c|c|c|}
\hline No. & Evaluation criteria & Points $\left(p_{i}\right)$ & Weights $\left(w_{i}\right)$ & $p_{i} \times w_{i}$ \\
\hline 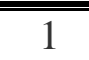 & Scale Accuracy & $\overline{00}$ & $\overline{22}$ & $\overline{00}$ \\
\hline 2 & Map Title & 2 & 1 & 2 \\
\hline 3 & North Arrow & 2 & 1 & 2 \\
\hline 4 & Scale & 2 & 2 & 4 \\
\hline 5 & legend & 1 & 1 & 1 \\
\hline 6 & Georeference & 1 & 2 & 2 \\
\hline 7 & Colour & 1 & 1 & 1 \\
\hline 8 & sheet Number & 0 & 1 & 0 \\
\hline 9 & Map Index & 1 & 1 & 1 \\
\hline 10 & Grid & 0 & 1 & 0 \\
\hline 11 & Overlapping & 1 & 1 & 1 \\
\hline 12 & Reliability & 1 & 2 & 2 \\
\hline 13 & Elevation & 0 & 1 & 0 \\
\hline 14 & Glossary & 0 & 1 & 0 \\
\hline 15 & $\begin{array}{l}\text { Conversion of } \\
\text { elevation }\end{array}$ & 0 & 1 & 0 \\
\hline 16 & $\begin{array}{l}\text { Magnetic } \\
\text { declination }\end{array}$ & 0 & 1 & 0 \\
\hline 17 & Lettering & 1 & 1 & 1 \\
\hline 18 & Source of data & 0 & 2 & 0 \\
\hline \multicolumn{3}{|c|}{ Sum } & 23 & 17 \\
\hline \multicolumn{2}{|c|}{$\begin{array}{c}\text { Weighted } \\
\text { Nagi Factor }=1 / 2 \times(17 / 23) \times 100 \% \\
\end{array}$} & \multicolumn{3}{|c|}{$37 \%$} \\
\hline
\end{tabular}




\section{CONCLUSION}

Although, map application is not bounded by a particular field, it may vary from taking reliable measurement, location determination, classification, interpretation, analysis etc. therefore, some map criteria such as scale and precision sometimes may appeared to be more important than the other criteria or vice versa.

This weighted Nagi method approach of map quality evaluation may solve the problem of map evaluation taking into account different weight for each criteria according to specific application.

Previous evaluation of Khartoum state survey authority map product using Nagi method provided 36\% satisfaction factor according to Sudan National Survey Authority Standards. This value increased to be $37 \%$ when applying the new weighted approach by doubling weight values of some criteria.

Choice between the two Nagi methods of numerical map quality evaluation depends on; are all map criteria have equal weights or not.

\section{REFERENCES}

[1]. Federal Geographic Data Committee (1998), Geospatial Positioning Accuracy Standards, Part 3 National Standard for Spatial Data Accuracy. Washington, D.C, Federal Geographic Data Committee Report.

[2]. Folks J. (1981), Horizontal Positional Accuracy, Ideas of Statistics.

[3]. Guptill, S.C. and J. L .Morrison (1995), Elements of spatial data quality, Oxford, pergamon.

[4]. http://is.muni.cz/do/rect/el/estud/prif/js11/fyz_chem/web/podpora/Definitions_of_Scientif ic_Terms.txt

[5]. Keates J.S. (1993), Cartographic Design and Production, New York, John Wily and Sons.

[6]. Menno Jan Kraak and Ferjan Ormeling (2010), Cartography Visualization of Geospatial Data, England, Pearson Education Limited.

[7]. Nagi Zomrawi, (2013), A New Approach of Map Quality Evaluation Applied to Khartoum State Survey Authority (Sudan), International journal of multidisciplinary sciences and engineering.

[8]. Nagi Zomrawi, (2011), The Effect of the Number of Control Points on the Adjusted Satellite Images, International journal of multidisciplinary sciences and engineering.

[9]. Rbinson, and others (1995), Element of Cartography, New York, John Wily and Sons.

[10]. S. Jabasingh and Suresh (2015), A New Approach of Map Quality Evaluation Applied to Tamil Nadu State Survey Authority (India), SSRG International Journal of Material Science and Engineering.

[11]. Wood, C. H. and C. P. keller, (1996), Cartographic Design: theoretical and practical perspectives, New York, John Wily and Sons. 